\title{
The prognostic value of the 8th edition of the American Joint Committee on Cancer (AJCC) staging system in triple-negative breast cancer
}

\author{
J. P. LI' ${ }^{1}$, X. M. ZHANG 2 , Y. S. ZHANG', L. H. ZHENG ${ }^{3}$, Y. J. LIU',* \\ ${ }^{1}$ Department of Breast Surgery, Fourth Hospital of Hebei Medical University, Shijiazhuang, Hebei, China; ${ }^{2}$ Research Center, Fourth Hospital \\ of Hebei Medical University, Shijiazhuang, Hebei, China; ${ }^{3}$ Department of Vascular Surgery, First Hospital of Hebei Medical University, \\ Shijiazhuang, Hebei, China
}

*Correspondence: lyj818326@126.com

Received January 7, 2019/Accepted February 27, 2019

\begin{abstract}
The American Joint Committee on Cancer (AJCC) released its 8th edition of cancer staging implemented in early 2018. This study aims to compare anatomic staging (AS) with prognostic staging (PS) based on the updated AJCC 8th edition staging manual. A retrospective single-center analysis of 313 triple-negative breast invasive ductal carcinoma patients who received surgery at department of breast surgery in the Fourth Hospital of Hebei Medical University from 01/2010 -12/2012 was performed. All cases were restaged using the AJCC 8th edition AS and PS system. The 7-year disease-free survival (DFS) and the 7-year overall survival (OS) rates were $76.30 \%$ and $78.27 \%$, respectively. Applying the PS system, $277(88.5 \%)$ patients of the AS groups were upstaged to the PS groups, 31 cases with IIIC and 5 cases with IV unchanged (11.5\%) and no cases downstaged. Both 7-year DFS and 7-year OS were significantly different in the different AS and PS groups (all, $\mathrm{p}<0.001$ ). The PS system was found to provide better prognostic information in patients with AS group IIB. A total of 43 patients with AS group IIB were upstaged by PS system, in which 30 patients were +2 upstaged to PS IIIB, and 13 patients were +3 upstaged to PS IIIC. PS IIIB and IIIC from AS IIB had significant differences in 7-year DFS $\left(\chi^{2}=5.628\right.$, $\mathrm{p}=0.014)$ and 7 -year OS $\left(\chi^{2}=6.037, \mathrm{p}=0.018\right)$. Both AS and PS systems proposed in the 8 th edition of the AJCC breast cancer staging manual had prognostic value in TNBC. Moreover, the PS system predicts clinical outcomes of TNBC patients more accurately than the traditional AS system.
\end{abstract}

Key words: American Joint Committee on Cancer, anatomic stage, prognostic stage, TNBC

The American Joint Committee on Cancer (AJCC) cancer staging system is used worldwide as an important tool for physicians to help predict disease progression and make therapeutic decisions. Anatomic factors, primary tumor (T), including regional lymph node involvement $(\mathrm{N})$ and distant metastases (M), have traditionally been the cornerstones for staging [1]. However, evolving knowledge of breast cancer biology and increased validation of various biomarkers for prognosis propound that specific biomarkers, such as estrogen receptor (ER), progesterone receptor ( $\mathrm{PgR})$, human epidermal growth factor receptor 2 (HER2), histological grade (HR) and so on, should be covered at the first diagnosis of breast cancer [2, 3]. Prognostic factors have become equally or even more important than anatomic factors to define prognosis, to determine the appropriate systemic therapy, and even to influence the selection of locoregional treatment. Accordingly, the updated AJCC 8th edition includes a dual stage designation for breast cancer: the traditional TNM-based anatomic stage (AS) system and more complex prognostic stage (PS) system. ER, PgR, HER2, HR and multigene assay are incorporated into the PS system [3].

After publication of the AJCC 8th edition staging manual in 2016 [4] and revision in December 2017 [5], the PS system was validated in various studies using single-institution cohorts or large population data base in different clinical settings (molecular subtypes, ethnic regions and population, and specific patients) [6-12]. Previous studies demonstrated the validity of PS compared to AS, finding that PS provided better prognostic information than AS. The updated 8th edition will require more validation for its clinical value in different population. To the best of our knowledge, there has not been any report describing the staging changes in triplenegative breast cancer (TNBC) specially. TNBC, accounting for approximately $12-17 \%$ of all breast cancers, is the most 
lethal subtype of breast cancer with low or no expression of ER, PgR, and HER2 [13]. For better survival estimate and appropriate systemic therapy of TNBC patients, it is necessary to identify and validate the new staging system in this subtype. Hence, we attempted to describe the staging changes by using the 8th edition of AJCC breast staging manual in a single-institution cohort of 313 TNBC patients in China, determining whether PS provide more refined prognostic stratification than AS for Asian TNBC.

\section{Patients and methods}

This retrospective study comprised 313 triple-negative breast invasive ductal carcinoma (IDC) patients who underwent primary surgery at Department of Breast Surgery in the Fourth Hospital of Hebei Medical University from January $1^{\text {st }}, 2010$ to December $31^{\text {st }}, 2012 ; 5$ patients with stage IV underwent palliative operation. Formalin-fixed, paraffinembedded specimens from 313 patients were retrieved and reassessed by examining hematoxylin and eosin-stained histologic sections. The histologic type of all the specimens was reconfirmed as breast invasive ductal carcinoma (IDC), according to the WHO classification. Patients were excluded if clinicopathological information were not available or incomplete (Figure 1). Appropriate adjuvant cytotoxic chemotherapy and radiation therapy after the surgery were conducted according to the standard guidelines. All patients were followed up after surgery until the date of death or October 2018. The study was approved by the institutional ethics committee of the Fourth Hospital of Hebei Medical University.

Clinicopathologic data. The molecular subtype of all the specimens was reconfirmed as TNBC, according to the 14 th St. Gallen International Expert Consensus. Two experienced pathologists reviewed all pathology specimens of 313 patients to determine the following tumor characteristics: histo- logic grade, tumor size, lympho-vascular invasion, tumor embolism, and immunohistochemical (IHC) staining for ER, PgR, HER2 and Ki67. Analyses for ER, PgR and HER2 were conducted according to the recommended guidelines of the American Society of Clinical Oncology and College of American Pathologists [14, 15]. The Ki67 index was scored as high when $30 \%$ or more of the tumor cells were expressing it [16]. Histologic grading (HG) was carried out using the Nottingham-combined histologic grade (Elston-Ellis modification of Scarff-Bloom-Richardson grading system). The baseline characteristics of the 313 patients in this study are reported in Table 1.

Anatomic and prognostic staging. All the enrolled cases were restaged using AS and PS system of the 8th edition AJCC staging manual [17]. AS system was based on the anatomic extent of cancer as defined by the anatomic $\mathrm{T}$, $\mathrm{N}$ and $\mathrm{M}$ categories. PS system took into account not only the information of anatomic parameters but also some the status of the prognostic biomarkers, including HER2, ER, $\mathrm{PR}$ and $\mathrm{HG}$.

Statistical analysis. Data was processed using SPSS for Windows (Version 22.0, IBM Corp., Armonk, NY, USA). Overall survival (OS) and disease-free survival (DFS) were calculated from the time of diagnosis to the time of event of interest, death from any cause, recurrence, or the final followup date. The associations between the survival and pathologic characteristics were examined using either Chi square statistical test or Fisher's exact test. Survival curves were plotted using the Kaplan-Meier method and the unstratified log-rank test was used to determine the significances of the survival differences between different subgroups. Cox proportional hazards model was used to estimate the hazard ratio (HR) of each clinicopathologic variables for OS and DFS. All predictors with $\mathrm{p}$-value $<0.05$ in univariate Cox analyses were used in multivariate analysis. $P$ values were two-tailed and considered significant when $<0.05$.

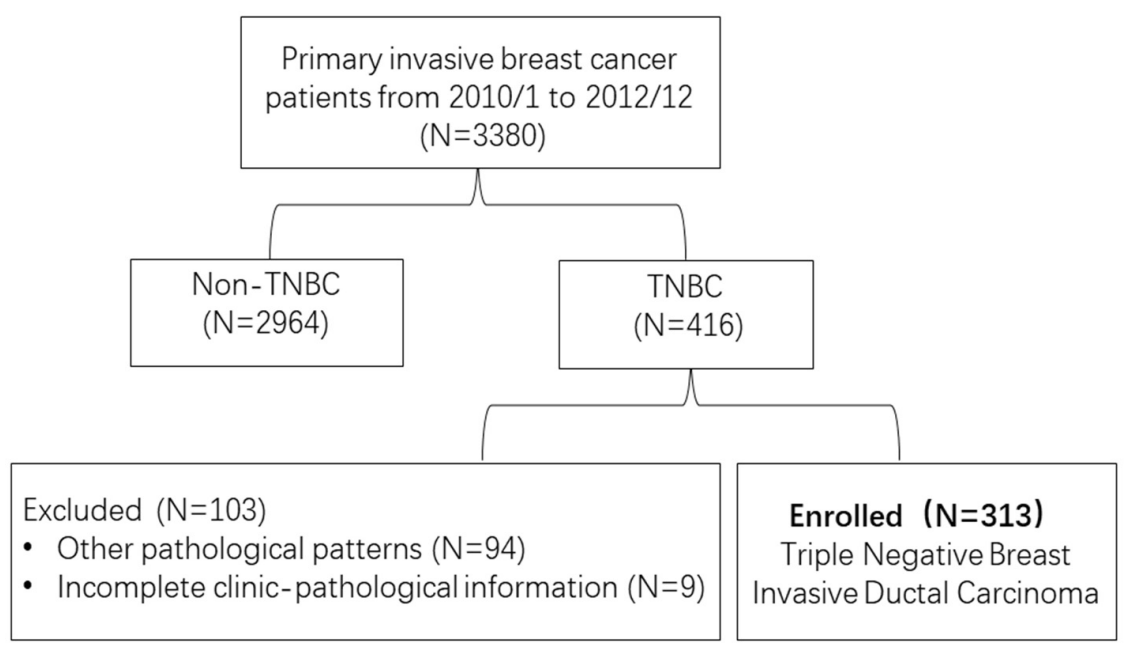

Figure 1. Flow diagram of the study cohort. 
Table 1. Clinicopathological characteristics of the patients with TNBC $(\mathrm{N}=313)$.

\begin{tabular}{|c|c|c|c|c|c|c|c|c|c|}
\hline \multirow{2}{*}{ Factor } & \multirow{2}{*}{$\begin{array}{c}\text { Cases } \\
(\mathrm{N})\end{array}$} & \multirow{2}{*}{$\begin{array}{l}\text { Cases of recurrence } \\
\text { and metastasis } \\
\qquad(\mathrm{N}=75)\end{array}$} & \multicolumn{3}{|c|}{ 7-year DFS } & \multirow{2}{*}{$\begin{array}{l}\text { Cases of } \\
\text { death } \\
(\mathrm{N}=68)\end{array}$} & \multicolumn{3}{|c|}{ 7-year OS } \\
\hline & & & $\begin{array}{c}\text { DFS } \\
(\%)\end{array}$ & $\chi^{2}$ & p-value & & $\begin{array}{l}\text { OS } \\
(\%)\end{array}$ & $\chi^{2}$ & p-value \\
\hline \multicolumn{10}{|l|}{ Age(year) } \\
\hline$\leq 35$ & 23 & 2 & $91.3 \%$ & 5.286 & 0.071 & 1 & $95.7 \%$ & 7.832 & 0.020 \\
\hline $36-64$ & 249 & 59 & $76.3 \%$ & & & 53 & $78.7 \%$ & & \\
\hline$\geq 65$ & 41 & 14 & $65.9 \%$ & & & 14 & $65.9 \%$ & & \\
\hline \multicolumn{10}{|l|}{ BMI $\left(\mathrm{kg} / \mathrm{m}^{2}\right)$} \\
\hline$<24.0$ & 147 & 36 & $75.7 \%$ & 0.562 & 0.755 & 32 & $78.2 \%$ & 0.150 & 0.928 \\
\hline $24.0-28.0$ & 106 & 23 & $78.3 \%$ & & & 22 & $79.2 \%$ & & \\
\hline$>28.0$ & 60 & 16 & $73.3 \%$ & & & 14 & $76.7 \%$ & & \\
\hline \multicolumn{10}{|l|}{ Menstrual status } \\
\hline Premenopausal & 149 & 32 & $78.5 \%$ & 0.964 & 0.326 & 27 & $81.9 \%$ & 2.173 & 0.170 \\
\hline Postmenopausal & 164 & 43 & $73.8 \%$ & & & 41 & $75.0 \%$ & & \\
\hline \multicolumn{10}{|l|}{ Breast surgery } \\
\hline BCS & 14 & 3 & $78.6 \%$ & 0.052 & 0.820 & 2 & $85.7 \%$ & 2.145 & 0.234 \\
\hline Mastectomy & 299 & 72 & $75.9 \%$ & & & 66 & $66.8 \%$ & & \\
\hline \multicolumn{10}{|l|}{ Tumor size } \\
\hline $\mathrm{T} 1$ & 175 & 25 & $85.7 \%$ & 31.468 & $<0.001$ & 21 & $88.1 \%$ & 35.262 & $<0.001$ \\
\hline $\mathrm{T} 2$ & 129 & 43 & $66.7 \%$ & & & 40 & $69.0 \%$ & & \\
\hline $\mathrm{T} 3$ & 5 & 3 & $40.0 \%$ & & & 3 & $40.0 \%$ & & \\
\hline $\mathrm{T} 4$ & 4 & 4 & $0 \%$ & & & 4 & $0 \%$ & & \\
\hline \multicolumn{10}{|c|}{ Lymph node status } \\
\hline No & 179 & 23 & $87.2 \%$ & 47.713 & $<0.001$ & 19 & $89.4 \%$ & 49.575 & $<0.001$ \\
\hline N1 & 76 & 21 & $72.4 \%$ & & & 20 & $73.7 \%$ & & \\
\hline $\mathrm{N} 2$ & 25 & 9 & $64.0 \%$ & & & 8 & $68.0 \%$ & & \\
\hline N3 & 33 & 22 & $33.3 \%$ & & & 21 & $36.4 \%$ & & \\
\hline \multicolumn{10}{|l|}{ Histological grade } \\
\hline I & 29 & 1 & $96.6 \%$ & 23.910 & $<0.001$ & 0 & $100 \%$ & 25.755 & $<0.001$ \\
\hline II & 204 & 40 & $80.4 \%$ & & & 36 & $82.4 \%$ & & \\
\hline III & 80 & 34 & $57.5 \%$ & & & 32 & $60.0 \%$ & & \\
\hline \multicolumn{10}{|c|}{ Lymph-vascular invasion } \\
\hline yes & 68 & 30 & $55.9 \%$ & 19.371 & $<0.001$ & 30 & $55.9 \%$ & 25.612 & $<0.001$ \\
\hline no & 245 & 45 & $81.6 \%$ & & & 38 & $84.5 \%$ & & \\
\hline \multicolumn{10}{|l|}{ Ki67 } \\
\hline$\leq 30 \%$ & 64 & 17 & $73.4 \%$ & 0.299 & 0.585 & 16 & $75.0 \%$ & 0.507 & 0.498 \\
\hline$>30 \%$ & 249 & 58 & $76.7 \%$ & & & 52 & $79.1 \%$ & & \\
\hline \multicolumn{10}{|l|}{ P53 } \\
\hline$\leq 10 \%$ & 130 & 23 & $82.3 \%$ & 4.797 & 0.029 & 20 & $84.6 \%$ & 5.257 & 0.022 \\
\hline$>10 \%$ & 183 & 52 & $71.6 \%$ & & & 48 & $73.8 \%$ & & \\
\hline
\end{tabular}

DFS: Disease-free survival; OS: Overall survival; TNBC: triple-negative breast cancer. BCS: breast conserving surgery. BMI: Body mass index. p-value: Chi square statistical test was used to estimate the survival differences between different subgroups.

\section{Results}

Patients' characteristics. In total, 3380 patients with primary invasive breast cancer were diagnosed and treated at Department of Breast Surgery in the Fourth Hospital of Hebei Medical University from January $1^{\text {st }}, 2010$ to December $31^{\text {st }}$, 2012. Among them, 416 (12.31\%) patients had the TNBC subtype. After exclusion of patients with special pathological types $(\mathrm{N}=94)$ and incomplete information $(\mathrm{N}=9), 313$ patients with triple-negative breast IDC were enrolled in this study. All patients were female and their median age was 54 (range, 23-80) years. A total of 149 (47.6\%) patients were pre-menopausal and 164 (52.4\%) were post-menopausal. The median follow-up time was 86 (range, 2-105) months. During the follow-up period, there were 73 cases categorized as anatomic stage I-III who had local recurrence and/ or distant metastasis, and 68 patients died at data cut-off (5 patients with stage IV included). The 7-year OS of all 313 
patients was $78.27 \%$ and the 7-year DFS of the 308 anatomic stage I-III patients was $76.30 \%$. The other clinic-pathological data are shown in Table 1.

Differences between the AS and PS system for TNBC. 313 enrolled TNBC patients were staged using the AS system of AJCC 8th edition. There were 121 patients (38.7\%) grouped as Stage I, 131 patients (41.9\%) grouped as Stage II, 56 patients (17.9\%) grouped as Stage III and 5 patients $(1.6 \%)$ grouped as Stage IV. When the same cohort was restaged by combined ER, PR, HER2 and HG status according to the PS system AJCC 8th edition, 277 cases (88.5\%) were upstaged, 31 cases with IIIC and 5 cases with IV remained unchanged (11.5\%) and no case was downstaged. After staging by the PS system, we found 122 patients $(39.0 \%)$ of our data grouped as Stage II, 186 patients (59.4\%) grouped as Stage III and 5 patients (1.6\%) grouped as Stage IV. The distribution of cases for AS and PS group is listed in Table 2.

Survival analyses of AS and PS groups. We analyzed patient survival using the log-rank test and found that there were significant differences for the 7-year DFS ( $\chi 2=13.897$, $\mathrm{p}<0.001)$ and 7 -year OS $(\chi 2=15.007, \mathrm{p}<0.001)$ in different AS (Table 3). Meanwhile, the different PS were significantly different with respect to the 7-year DFS $(\chi 2=27.380, \mathrm{p}<0.001)$ and 7 -year OS $\left(\chi^{2}=27.904, p<0.001\right.$, Table 3$)$. The Kaplan-
Meier curves for the different AS groups and PS groups are presented in Figure 2.

After the PS group changes were made in each AS group, the groups were compared with the Log-rank test. A statistically significant deference was found in the AS group IIB (Table 4). A total of 43 patients with AS group IIB was upstaged by PS system, in which 30 patients were +2 upstaged to PS IIIB, and 13 patients were +3 upstaged to PS IIIC. The 7 -years

Table 2. Differences of stages when changed from AS to PS groups.

\begin{tabular}{lccccc}
\hline \multirow{2}{*}{ AS Groups } & & \multicolumn{4}{c}{ PS Groups } \\
\cline { 2 - 6 } & & \multicolumn{2}{c}{ Unchanged } & \multicolumn{2}{c}{ Upstaged } \\
\hline Stage & Cases (N) & Stage & Cases (N) & Stage & Cases (N) \\
I & 121 & I & 0 & IIA & 121 \\
IIA & 88 & IIA & 0 & IIB & 1 \\
& & & & IIIA & 87 \\
IIB & 43 & IIB & 0 & IIIB & 30 \\
& & & & IIIC & 13 \\
IIIA & 23 & IIIA & 0 & IIIC & 23 \\
IIIB & 2 & IIIB & 0 & IIIC & 2 \\
IIIC & 31 & IIIC & 31 & & \\
IV & 5 & IV & 5 & & \\
\hline
\end{tabular}

AS: Anatomic Stage. PS: Prognostic Stage.
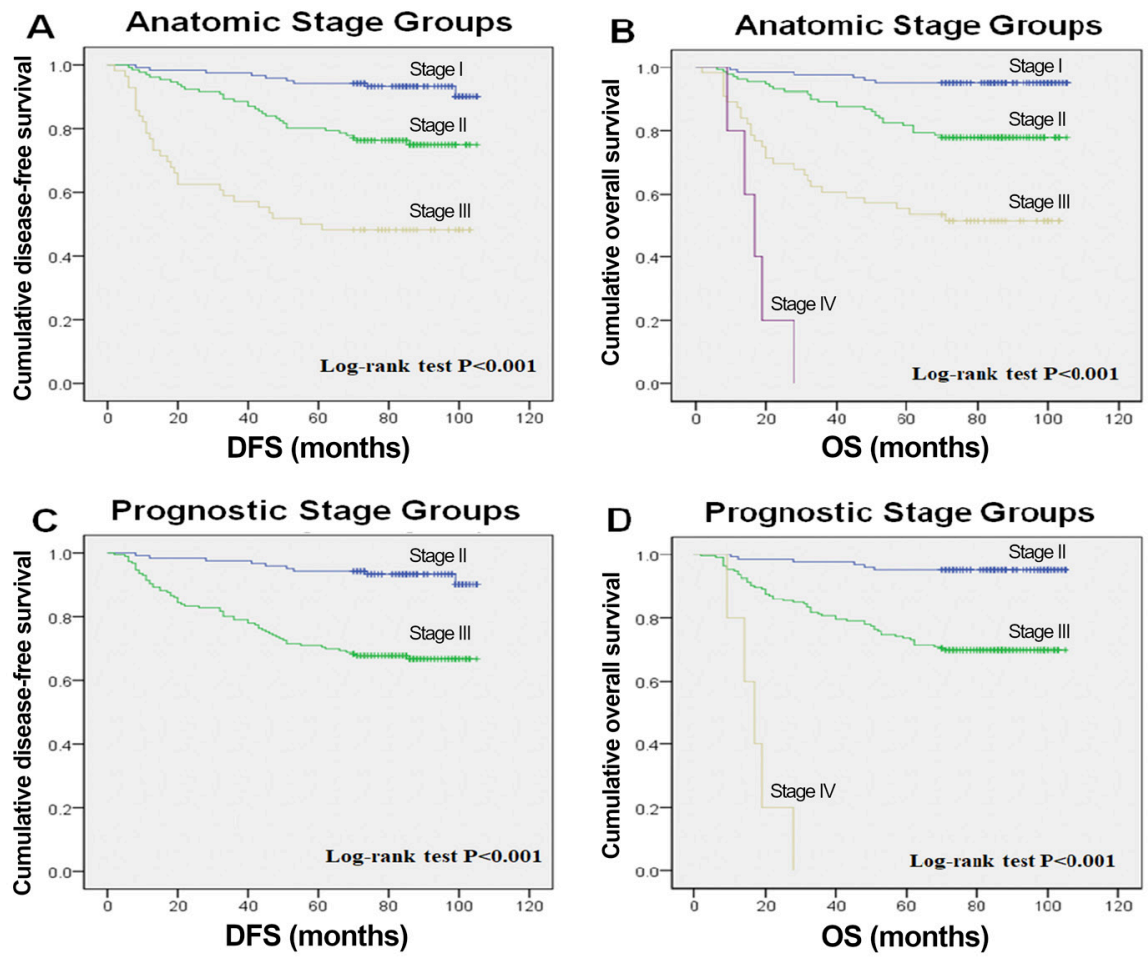

Figure 2. DFS and OS of different anatomic stage (AS) groups and prognostic stage (PS) groups (N=313). A) Disease-free survival (DFS) and B) overall survival (OS) for AS groups; there were significant differences in DFS $\left(\chi^{2}=16.752, p<0.001\right)$ and $0 S\left(\chi^{2}=25.038, p<0.001\right)$ between anatomic stage groups. C) Disease-free survival (DFS) and D) overall survival (OS) for PS groups; there were significant differences in DFS $\left(\chi^{2}=16.752\right.$, p $\left.<0.001\right)$ and OS $\left(\chi^{2}=25.038, p<0.001\right)$ between prognostic stage groups. 
DFS/OS was $69.8 \%$ in patients with AS IIB. In a deep analysis of AS IIB patients, the 7-years DFS/OS in patients with +2 upstaged PS IIIB was different with the rate of patients with +3 upstaged PS IIIC significantly ( $80.0 \%$ vs. $46.2 \%)$. KaplanMeier curve showed that PS IIIB and IIIC from AS IIB had significant differences in 7-year DFS $(\chi 2=5.628, \mathrm{P}=0.014)$ and 7 -year OS ( $\chi 2=6.037, \mathrm{P}=0.018$ ) (Figure 3 ). The PS system was found to provide better prognostic information in patients with AS IIB.

Univariate and multivariate analyses in Cox regression model. Cox proportional hazards model was used to estimate specific clinicopathologic variables for OS and DFS. Univariate Cox analysis of clinicopathological characteristics indicated that T (2-4), N (1-3), G3, p53 and with lymphvascular invasion was significantly associated with worse DFS ( $<<0.05$; Table 5). Multivariate Cox analysis of specific important markers and all the predictors with $\mathrm{p}$-values $<0.05$ in univariate Cox analyses indicated that T4 was an independent prognostic factor for bad DFS (HR: 11.832; 95\%CI: 3.464-40.419; $\mathrm{p}<0.001$, Table 5), as well as N3 (HR: 3.973; 95\%CI: 1.901-8.302; $<<0.001$, Table 5). Similarly, T (2-4), N $(1-3)$, age $(\geq 65 y), p 53$ and with lymph-vascular invasion was significantly associated with worse OS $(\mathrm{p}<0.05$; Table 6$)$ in the univariate analysis. The multivariate analysis showed that $\mathrm{T}(2-4), \mathrm{N}(1-3)$ and p53 appeared to be independent risk factors for poor OS (Table 6).

Table 3. Comparison of DFS and OS using the 8th edition of AJCC anatomic and prognostic staging system of TNBC (N=313).

\begin{tabular}{|c|c|c|c|c|c|c|c|c|c|c|}
\hline \multirow{2}{*}{$\begin{array}{l}\text { Staging } \\
\text { system }\end{array}$} & \multirow{2}{*}{ Stage } & \multirow{2}{*}{$\begin{array}{c}\text { Cases } \\
(\mathrm{N}=313)\end{array}$} & \multirow{2}{*}{$\begin{array}{l}\text { Events } \\
(\mathrm{N}=75)\end{array}$} & \multirow{2}{*}{ Percentage } & \multicolumn{2}{|c|}{ DFS } & \multirow{2}{*}{$\begin{array}{l}\text { Deaths } \\
(\mathrm{N}=68)\end{array}$} & \multirow{2}{*}{ Percentage } & \multicolumn{2}{|c|}{ OS } \\
\hline & & & & & $\chi^{2}$ & p-value & & & $\chi^{2}$ & p-value \\
\hline \multirow{4}{*}{$\begin{array}{l}\text { Anatomic } \\
\text { staging system } \\
\text { (AS) }\end{array}$} & I & 121 & 9 & $92.6 \%$ & 43.247 & $<0.001$ & 7 & $94.2 \%$ & 59.214 & $<0.001$ \\
\hline & II & 131 & 32 & $75.6 \%$ & & & 29 & $77.9 \%$ & & \\
\hline & III & 56 & 29 & $48.2 \%$ & & & 27 & $51.8 \%$ & & \\
\hline & IV & 5 & & & & & 5 & $0 \%$ & & \\
\hline \multirow{3}{*}{$\begin{array}{l}\text { Prognostic } \\
\text { staging system } \\
\text { (PS) }\end{array}$} & II & 122 & 9 & $92.6 \%$ & 27.380 & $<0.001$ & 7 & $94.3 \%$ & 27.904 & $<0.001$ \\
\hline & III & 186 & 61 & $67.2 \%$ & & & 56 & $69.9 \%$ & & \\
\hline & IV & 5 & & & & & 5 & $0 \%$ & & \\
\hline
\end{tabular}

Events: Including local recurrence and/or distant metastasis; p-value: Log-rank test was used to estimate the survival differences between different subgroups. DFS: Disease-free survival; OS: Overall survival; AJCC: American Joint Committee on Cancer.

Table 4. The effects of alterations on survival after reclassification of AS to PS system.

\begin{tabular}{|c|c|c|c|c|c|c|c|c|c|c|c|}
\hline \multicolumn{2}{|l|}{ AS } & \multicolumn{2}{|l|}{ PS } & \multicolumn{4}{|c|}{ 7-year DFS } & \multicolumn{4}{|c|}{ 7-year OS } \\
\hline Stage & $\mathbf{N}$ & Alteration & $\mathbf{N}$ & Events $(\mathrm{N})$ & DFS (\%) & $\chi^{2}$ & p-value & Death $(\mathrm{N})$ & OS (\%) & $\chi^{2}$ & p-value \\
\hline IIB & 43 & $(\mathrm{AS}) \mathrm{IIB} \rightarrow(\mathrm{PS})$ IIIB & 30 & 6 & $80 \%$ & 5.628 & 0.014 & 6 & $80 \%$ & 6.037 & 0.018 \\
\hline & & $(\mathrm{AS}) \mathrm{IIB} \rightarrow(\mathrm{PS})$ IIIC & 13 & 7 & $46.2 \%$ & & & 7 & $46.2 \%$ & & \\
\hline
\end{tabular}

DFS - disease free survival; DSS - disease specific survival; p-value: Log-rank test.
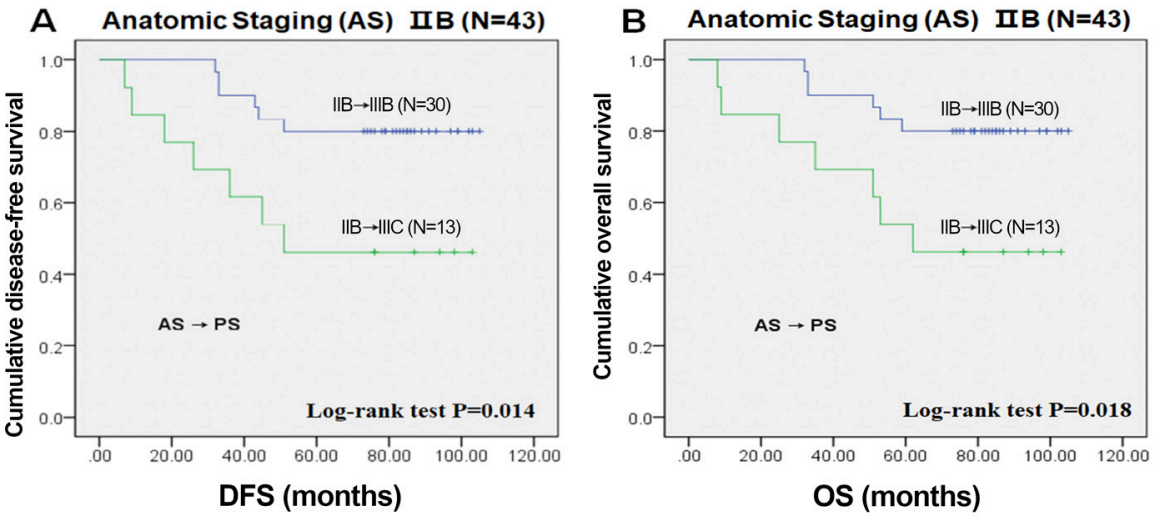

Figure 3. Survival analysis of anatomic stage IIB patients. A) Disease-free survival (DFS) and (B) overall survival (OS); there were significant differences in DFS $\left(\chi^{2}=5.628, p=0.014\right)$ and OS $\left(\chi^{2}=6.037, p=0.018\right)$ between PS IIIB group and PS IIIC group after reclassification from AS IIB in DFS and OS according to pathologic prognostic stages. 
Table 5. Univariate and multivariate analyses (Cox regression) for DFS.

\begin{tabular}{|c|c|c|c|c|c|c|}
\hline \multirow{2}{*}{ Variable } & \multicolumn{3}{|c|}{ Univariate analysis for DFS } & \multicolumn{3}{|c|}{ Multivariate analysis for DFS } \\
\hline & HR & $95 \% \mathrm{CI}$ & p-value & HR & $95 \% \mathrm{CI}$ & p-value \\
\hline $\mathrm{T} 2$ & 2.632 & $1.606-4.314$ & $<0.001$ & 1.571 & $0.929-2.656$ & 0.092 \\
\hline T3 & 6.233 & $1.876-20.709$ & 0.003 & $3 . .011$ & $0.826-10.976$ & 0.095 \\
\hline $\mathrm{T} 4$ & 40.694 & $13.265-124.843$ & $<0.001$ & 11.832 & $3.464-40.419$ & $<0.001$ \\
\hline N1 & 2.381 & $1.318-4.304$ & 0.004 & 1.790 & $0.966-3.317$ & 0.064 \\
\hline $\mathrm{N} 2$ & 3.526 & $1.631-7.623$ & 0.001 & 1.982 & $0.867-4.535$ & 0.105 \\
\hline N3 & 8.981 & $4.983-16.185$ & $<0.001$ & 3.973 & $1.901-8.302$ & $<0.001$ \\
\hline G2 & 6.239 & $0.858-45.387$ & 0.071 & 2.976 & $0.394-22.444$ & 0.290 \\
\hline G3 & 16.205 & $2.218-118.421$ & 0.006 & 5.060 & $0.646-39.626$ & 0.123 \\
\hline Lymph-vascular invasion & 2.867 & $1.805-4.555$ & $<0.001$ & 1.318 & $0.766-2.268$ & 0.319 \\
\hline P53 & 1.693 & $1.036-2.767$ & 0.035 & 1.564 & $0.945-2.588$ & 0.082 \\
\hline
\end{tabular}

HR: hazard ratio. CI: confidence interval.

Table 6. Univariate and multivariate analyses (Cox regression) for OS.

\begin{tabular}{|c|c|c|c|c|c|c|}
\hline \multirow{2}{*}{ Variable } & \multicolumn{3}{|c|}{ Univariate analysis for OS } & \multicolumn{3}{|c|}{ Multivariate analysis for OS } \\
\hline & HR & $95 \% \mathrm{CI}$ & p-value & HR & 95\% CI & p-value \\
\hline $\mathrm{T} 2$ & 2.925 & $1.709-5.003$ & $<0.001$ & 1.752 & $1.042-2.946$ & 0.034 \\
\hline T3 & 7.564 & $2.242-25.516$ & 0.001 & 3.635 & $1.013-13.043$ & 0.048 \\
\hline $\mathrm{T} 4$ & 31.180 & $10.237-94.964$ & $<0.001$ & 13.957 & $4.127-47.201$ & $<0.001$ \\
\hline N1 & 2.855 & $1.510-5.398$ & 0.001 & 1.967 & $1.062-3.640$ & 0.031 \\
\hline $\mathrm{N} 2$ & 3.833 & $1.666-8.818$ & 0.002 & 2.553 & $1.140-5.717$ & 0.023 \\
\hline N3 & 9.864 & $5.237-18.581$ & $<0.001$ & 4.728 & $2.278-9.812$ & $<0.001$ \\
\hline $\operatorname{Age}(\geq 65 y)$ & 8.259 & $1.080-63.136$ & 0.042 & 3.013 & $0.665-13.661$ & 0.153 \\
\hline Lymph-vascular invasion & 3.351 & $2.069-5.429$ & $<0.001$ & 1.418 & $0.824-2.443$ & 0.207 \\
\hline P53 & 1.812 & $1.073-3.058$ & 0.026 & 1.683 & $1.020-2.775$ & 0.041 \\
\hline
\end{tabular}

HR: hazard ratio. CI: confidence interval.

\section{Discussion}

Anatomic factors have always been at the foundation of AJCC staging. $\mathrm{T}$ and $\mathrm{N}$ are demonstrated to be independent risk factors for survival in our multivariate analysis. However, the value for prognosis has faced a challenge from the emerging importance of prognostic biomarkers such as ER, PR, HER2, HG and gene expression prognostic panels in breast cancer $[2,3]$. With the rapid evolution of knowledge in cancer molecular underpinnings and the validation of aforementioned biologic factors can predict cancer outcomes and response to treatment with better accuracy, a multidisciplinary team of breast cancer experts recognized the need to incorporate biologic factors into the TNM staging system, thus establishing the update 8th edition AJCC staging manual [17]. It deserves to be mentioned that p53, a novel biomarker for TNBC, appeared to be an independent risk factor for poor OS in our study.

At the end of 2016, the initial version of the AJCC Cancer Staging Manual 8th Edition was published [4]. The 8th edition incorporated HG and multigene assay findings, as well as ER, PR and HER2, providing PS system and AS system. However, in the initial version of the 8 th edition,
PS group could not be assigned in some specific patients. A study conducted by the MD Anderson Cancer Center with a cohort of 3327 patients reported that $13.6 \%$ of patients could not be assigned to PS groups [9]. Then, the revised 8th edition of AJCC cancer staging was applied in December 2017 [5]. Up to now, various studies in different clinical settings have validated that the PS system provides better prognostic information than the AS system [6-12, 18-21]. The rates of staging changes (upstage/downstage) from AS to PS ranged from $41 \%$ to $68.8 \%$, because of different single/ multi institution study, different sample, different race and age and different subtype proportions.

Zhou et al. [6] analyzed the prognostic value of AJCC 8th edition Cancer Staging System in HER2-enriched breast cancer. 117 cases $(68.8 \%)$ of AS groups had been restaged to PS groups in 170 HER2-positive breast cancer patients. They concluded that both AS and PS groups in the 8th edition of AJCC had prognostic value in HER2-enriched subtype breast cancer. Ye et al. [7] aimed to make a prognostic evaluation in luminal-A breast cancer by using AJCC 8th Edition. They reported that $41.6 \%$ of the patients changed from AS to PS groups in a cohort of 412 luminal-A breast cancer patients. They found the significant difference between 
different PS groups in DFS and OS rates, but no difference was found between different AS groups in OS. Xu et al. [8] found that there were 372 cases (46.7\%) assigned to a different group in 796 patients with luminal B HER-negative breast cancer. They found that the PS system refined the AS group in luminal B HER2-negative breast cancer. These three abovementioned studies were conducted merely in single institution. Weiss et al. [9] validated that the PS gave better prognostic information than the AS in both a singleinstitution cohort (3327 patients with stage I-IIIC breast cancer in MD Anderson) and a large population database (54727 patients with stage I to IV from California Cancer Registry). Abdel-Rahman [10] reported that the PS system showed an improvement in determination of prognosis when compared with the AS system in 209304 patients with non-metastatic breast cancer from the SEER database (2010-2014). Targeting at specific groups, Wang et al. [11] evaluated the PS system in locally advanced breast cancer (LABC) based on the SEER 18 database. They reported that PS provided accurate prognostic information for LABC compared with anatomic TNM stage. In addition, Joo et al. [12] evaluated PS of 8th edition AJCC staging manual in patients with internal mammary lymph node (IMN) metastasized breast cancer. Despite the small number of patients $(\mathrm{N}=114)$, the prognostic stage provided accurate information for IMN metastasized breast cancer.

Patients with breast cancer expressing ER, PR or HER2 were downstaged or left unchanged by the PS system [18]. Conversely, patients with TNBC were upstaged or left unchanged in the reclassification. TNBC is the only molecular subtype in which downstage was inexistent [17]. To the best of our knowledge, there has not been any report describing the staging changes in TNBC specially. Herein, we conducted a retrospective study and survival analysis of the AS and PS groups according to the 8th edition of the AJCC cancer staging system in TNBC. Our study is the first to describe the staging changes of TNBC in low-to middleincome countries (LMCs). Our study demonstrated that there were marked staging changes (rate of upstage: $88.50 \%$ ) when AS and PS system were used in TNBC. We found that PS groups provided stratified prognostication within the same AS IIB group. A total of 43 patients with AS group IIB were upstaged by PS system, in which 30 patients were +2 upstaged to PS IIIB, and 13 patients were +3 upstaged to PS IIIC. PS IIIB and IIIC from AS IIB had significant differences in DFS $\left(\chi^{2}=5.628, p=0.014\right)$ and OS $\left(\chi^{2}=6.037\right.$, $\mathrm{p}=0.018$ ). Ibis et al. [22] also found patients with AS IIB breast cancer restaged to different PS groups in a cohort of 353 Turkish patients, with a significant difference in 10-year DFS among restaged PS groups. Thus, we confirmed that PS system provided more accurate information on prognosis than AS system in patients with TNBC, especially in AS IIB group. This conclusion concurred with the subgroup analysis of another one-institution study in different molecular subtypes of breast cancer from South Korea [23]. Recently, they reported the PS system showed difference in terms of OS and DFS in TNBC ( $p=0.008$ and $p=0.03$ ), but different AS groups did not demonstrate significant survival discrimination ( $\mathrm{p}=0.237$ and $\mathrm{p}=0.697$ ) [23]. On the contrary, we found that both AS and PS systems had prognostic value in terms of OS and DFS ( $\mathrm{p}<0.001)$ with a twice size of TNBC sample (313 cases vs. 141 cases). This divergence may be due to the different population and stage proportions.

The limitations of this study should be considered. First, it is primarily a retrospective study and the number of Asian TNBC patients is relatively limited in a single-institution. Next, the little number of AS IIIB cases $(\mathrm{N}=2)$ was not adequate to analyze deeply when the PS system was applied. Finally, we did not distinguish IA and IB because of few information of $\mathrm{pN} 1 \mathrm{mi}$ in archived file. Validation studies should be done with multi-institution, prospective studies with large samples and long follow-up timed.

In conclusion, this is the first study to describe staging changes by using AS and PS systems of 8th AJCC staging manual in Chinese TNBC patients. There was a high incidence of staging changes (88.5\%) from AS to PS groups. PS system provided more accurate information on prognosis than AS system in patients with TNBC, especially in AS IIB group. We should follow the updated manual to make precise diagnoses, to predict the risk accurately, to select better individualized therapy, and therefore give better survival outcomes. The 8th AJCC cancer staging manual will remain the worldwide standard for breast cancer staging.

\section{References}

[1] CSERNI G, CHMIELIK E, CSERNI B, TOT T. The new TNM-based staging of breast cancer. Virchows Arch 2018; 472: 697-703. https://doi.org/10.1007/s00428-018-2301-9

[2] AMIN MB, GREENE FL, EDGE SB, COMPTON CC, GERSHENWALD JE et al. The Eighth Edition AJCC Cancer Staging Manual: Continuing to build a bridge from a populationbased to a more "personalized" approach to cancer staging. CA Cancer J Clin 2017; 67: 93-99. https://doi.org/10.3322/ caac. 21388

[3] GiUliano AE, CONNOlly JL, EDGE SB, MitTENDORF EA, RUGO HS et al. Breast Cancer-Major changes in the American Joint Committee on Cancer eighth edition cancer staging manual. CA Cancer J Clin 2017; 67: 290-303. https://doi.org/10.3322/caac.21393

[4] HORTOBAGYI G, CONNOLLY JL, D'ORSI CJ, EDGE SB, MITTENDORF EA et al. Breast, pp 587-628. In: MB Amin, SB Edge, FL Greene, DR Byrd, RK Brookland et al (Eds.). AJCC cancer staging manual, 8th Edition. Springer, New York 2017, p 1032. ISBN 978-3-319-40617-6.

[5] American Joint Committee on Cancer. Updated breast chapter for 8th edition. https://cancerstaging.org/referencestools/deskreferences/Pages/Breast-Cancer-Staging.aspx Accessed 25 January 2018. 
[6] ZHOU B, XU L, YE J, XIN L, DUAN X et al. The prognostic value of the 8th edition of the American Joint Committee on Cancer (AJCC) staging system in HER2-enriched subtype breast cancer, a retrospective analysis. Anticancer Res 2017; 37: 4615-4621. https://doi.org/10.21873/anticanres.11862

[7] YE J, WANG W, XU L, DUAN X, CHENG Y et al. A retrospective prognostic evaluation analysis using the 8th edition of American Joint Committee on Cancer (AJCC) cancer staging system for luminal A breast cancer. Chin J Cancer Res 2017; 29: 351-360. https://doi.org/10.21147/j.issn.10009604.2017.04.08

[8] XU L, LI JH, YE JM, DUAN XN, CHENG YJ et al. A Retrospective Survival Analysis of Anatomic and Prognostic Stage Group Based on the American Joint Committee on Cancer 8th Edition Cancer Staging Manual in Luminal B Human Epidermal Growth Factor Receptor 2-negative Breast Cancer. Chin Med J (Engl) 2017; 130: 1945-1952. https://doi. org/10.4103/0366-6999.211896

[9] WEISS A, CHAVEZ-MACGREGOR M, LICHTENSZTAJN DY, YI M, TADROS A et al. Validation study of the American Joint Committee on Cancer 8th edition prognostic stage compared with the anatomic stage in breast cancer. JAMA Oncol 2018; 4: 203-209. https://doi.org/10.1001/jamaoncol.2017.4298

[10] ABDEL-RAHMAN O: Validation of the 8th AJCC prognostic staging system for breast cancer in a population-based setting. Breast Cancer Res Treat 2018; 168: 269-275. https:// doi.org/10.1007/s10549-017-4577-x

[11] WANG M, CHEN H, WU K, DING A, ZHANG M et al. Evaluation of the prognostic stage in the 8th edition of the American Joint Committee on Cancer in locally advanced breast cancer: An analysis based on SEER 18 database. Breast 2018; 37: 56-63. https://doi.org/10.1016/j.breast.2017.10.011

[12] JOO JH, KIM SS, SON BH, DO AHN S, JUNG JH et al. Evaluation of the Prognostic Stage in the 8th Edition of the American Joint Committee on Cancer in Patients with Breast Cancer and Internal Mammary Lymph Node Metastasis. Anticancer Res 2018; 38: 5357-5361. https://doi. org/10.21873/anticanres.12864

[13] FOULKES WD, SMITH IE, REIS-FILHO JS. Triple-negative breast cancer. N Engl J Med 2010; 363: 1938-1948. https:// doi.org/10.1056/NEJMra1001389

[14] HAMMOND ME, HAYES DF, DOWSETT M, ALLRED DC, HAGERTY KL et al. American Society of Clinical Oncology/ College of American Pathologists guideline recommendations for immunohistochemical testing of estrogen and progesterone receptors in breast cancer (unabridged version). Arch Pathol Lab Med 2010; 134: e48-72. https://doi. org/10.1043/1543-2165-134.7.e48
[15] WOLFF AC, HAMMOND ME, HICKS DG, DOWSETT M, MCSHANE LM et al. Recommendations for human epidermal growth factor receptor 2 testing in breast cancer: American Society of Clinical Oncology/College of American Pathologists clinical practice guideline update. Arch Pathol Lab Med 2014; 138: 241-256. https://doi.org/10.5858/arpa.20130953-SA

[16] YERUSHALMI R, WOODS R, RAVDIN PM, HAYES MM, GELMON KA. Ki67 in breast cancer: prognostic and predictive potential. Lancet Oncol 2010; 11: 174-183. https://doi. org/10.1016/S1470-2045(09)70262-1

[17] GIULIANO A E, EDGE S B, HORTOBAGYI G N. Eighth Edition of the AJCC Cancer Staging Manual: Breast Cancer. Ann Surg Oncol 2018; 25: 1783-1785. https://doi. org/10.1245/s10434-018-6486-6

[18] PLICHTA JK, REN Y, THOMAS SM, GREENUP RA, FAYANJU OM et al. Implications for Breast Cancer Restaging Based on the 8th Edition AJCC Staging Manual. Ann Surg 2018. https://doi.org/10.1097/SLA.0000000000003071

[19] LI X, ZHANG Y, MEISEL J, JIANG R, BEHERA M et al. Validation of the newly proposed American Joint Committee on Cancer (AJCC) breast cancer prognostic staging group and proposing a new staging system using the National Cancer Database. Breast Cancer Res Treat 2018; 171: 303-313. https://doi.org/10.1007/s10549-018-4832-9

[20] KIM JY, LIM JE, JUNG HH, CHO SY, CHO EY et al. Validation of the new AJCC eighth edition of the TNM classification for breast cancer with a single-center breast cancer cohort. Breast Cancer Res Treat 2018; 171: 737-745. https:// doi.org/10.1007/s10549-018-4858-Z

[21] WONG RX, WONG FY, LIM J, LIAN WX, YAP YS. Validation of the AJCC 8th prognostic system for breast cancer in an Asian healthcare setting. Breast 2018; 40: 38-44. https:// doi.org/10.1016/j.breast.2018.04.013

[22] IBIS K, OZKURT S, KUCUCUK S, YAVUZ E, SAIP P. Comparison of Pathological Prognostic Stage and Anatomic Stage Groups According to the Updated Version of the American Joint Committee on Cancer (AJCC) Breast Cancer Staging 8th Edition. Med Sci Monit 2018; 24: 3637-3643. https://doi. org/10.12659/MSM.911022

[23] JANG N, CHOI JE, KANG SH, BAE YK. Validation of the pathological prognostic staging system proposed in the revised eighth edition of the AJCC staging manual in different molecular subtypes of breast cancer. Virchows Arch 2019; 474: 193-200. https://doi.org/10.1007/s00428-018-2495-x 